\title{
Erratum: Brian et al. (2019)
}

In the article Brian, A., Taunton, S., Shortt, C., Pennell, A., \& Sacko, R. (2019). Predictors of physical activity for preschool children with and without disabilities from socioeconomically disadvantaged settings. Adapted Physical Activity Quarterly, 36(1), 77-90, doi:10.1123/apaq.2017-0191, the author Ryan Sacko's affiliation was listed incorrectly in the footnote on the first page as the University of South Carolina. Sacko is with The Citadel in Charleston, SC. The online version of this article has been corrected. We apologize for this error. 